\title{
The variable communicating branches between musculocutaneous and median nerves: a morphological study with clinical implications
}

\author{
El Falougy H, Selmeciova P, Kubikova E, Stenova J, Haviarova Z \\ Institute of Anatomy, Faculty of Medicine, Comenius University, Bratislava, Slovakia. \\ hisham.elfalougy@fmed.uniba.sk
}

\begin{abstract}
Background: The course of the brachial plexus, its relations with surrounding structures and unique primary and secondary divisions result in its wide range of anatomical variations. Most of these variations were detected during anatomical dissections and studies. It has been found that $53 \%$ of studied brachial plexuses contained variations. The communication between musculocutaneous and median nerves is the most common variation of infraclavicular part of brachial plexus.

Methods: During gross anatomical dissections of peripheral nerves, we observed neuronatomical variations in upper limbs of four formalin embalmed adult cadavers. Musculocutaneous and median nerves were connected by a communicating branch at distinct level in each cadaver. The formation and relations of both nerves were noted in each case to exclude the existence of other anatomical variations. The connections were measured and documented by digital camera.

Results: The communicating fibers of variations 1 and 2 were located in the upper third of arm and proximally to musculocutaneous nerve penetration through coracobrachialis muscle. In variations 3 and 4, the communicating branch was situated in the lower third of arm and distal to the nerve penetration point.

Conclusion: Variable interconnections between musculocutaneous and median nerve have to be considered in diagnosis of nerve lesions in axillary and arm regions. Compound musculocutaneous and median nerve neuropathy would occur in lesions of the interconnecting branches. Injuries of musculocutaneous nerve proximal to these branches can cause particular and unexpected symptoms, such as weakness of forearm flexors and thenar muscles (Fig. 6, Ref. 28). Full Text in PDF www.elis.sk.

Key words: musculocutaneous nerve, median nerve, interconnection, variation.
\end{abstract}

The brachial plexus is formed by the anterior rami of the cervical nerves $\mathrm{C} 5-\mathrm{C} 8$. It receives variable connections from the anterior rami of the fourth cervical nerve (C4) and the first thoracic nerve (T1). The prefixed type of the plexus is characterized by thick contributive nerve fibers from $\mathrm{C} 4$ and thin or absent fibers from $\mathrm{T} 1$. In the constitution of the postfixed brachial plexus the first two thoracic nerves (T1-T2) with absence of the nerve connection from $\mathrm{C} 4$ take part. The prefixation of the plexus is more common than its postfixation $(1,2)$.

The roots of the plexus lie in the posterior cervical triangle between the anterior and middle scalene muscles. The nerve roots unite to form complex nerve network, from which the three primary trunks of the brachial plexus are branching: superior middle and inferior trunks. These are passing together with the subclavian artery under the clavicle and through the scalene gap. Each trunk is divided into anterior and posterior divisions. The lateral, medial and posterior cords of the plexus are formed by these divisions in

Institute of Anatomy, Faculty of Medicine, Comenius University, Bratislava, Slovakia

Address for correspondence: Hisham El Falougy, MD, PhD, Institute of Anatomy, Faculty of Medicine, Comenius University, Spitalska 24, SK81372 Bratislava, Slovakia.

Phone: +421.2 .59357374$ the axillary fossa. The cords are named according to their relative position around the axillary artery $(3,4)$.

From the topographical point of view the plexus is divided into supraclavicular and infraclavicular parts. The supraclavicular part gives off branches to innervate the shoulder girdle regions. The infraclavicular part is giving the nerve supply mainly for the free upper limb (4).

The course of the brachial plexus, its relations with surrounding structures and the unique primary and secondary divisions result in its wide range of anatomical variations. Most of these variations were detected during the anatomical dissections and studies. It has been found that $53 \%$ of studied brachial plexuses contained significant anatomical variations (3). The most common variations are in the root formation of the plexus. The prefixed type is found to make $28-62 \%$ of anatomical variations of the plexus against $16-73 \%$ for the postfixed type (5).

The brachial plexus is involved in many syndromes and disorders throughout its course. In the thoracic outlet syndrome the neurovascular bundle containing the brachial plexus and the subclavian artery is compressed between the anterior and middle scalene muscles. The carpal tunnel syndrome is the result of the median nerve compression. The injuries of the brachial plexus can be classified as traumatic or obstetric. The traction injuries present 
$95 \%$ of cases $(6,7)$. The diagnosis and treatment of these disorders should be based on the knowledge of normal anatomy and variations of the brachial plexus and its topographical relations.

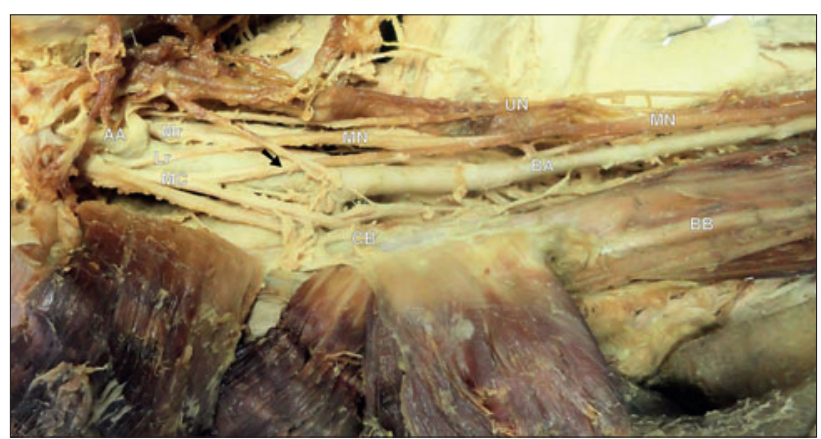

Fig. 1. Unilateral proximal communicating branch (black arrow) between musculocutaneous (MC) and median (MN) nerves in male adult cadaver. $\mathrm{Mr}$ - medial root of median nerve, $\mathrm{Lr}$ - lateral root of median nerve, UN - ulnar nerve, AA - axillary artery, BA - brachial artery, CB - coracobrachialis, BB - biceps brachii.
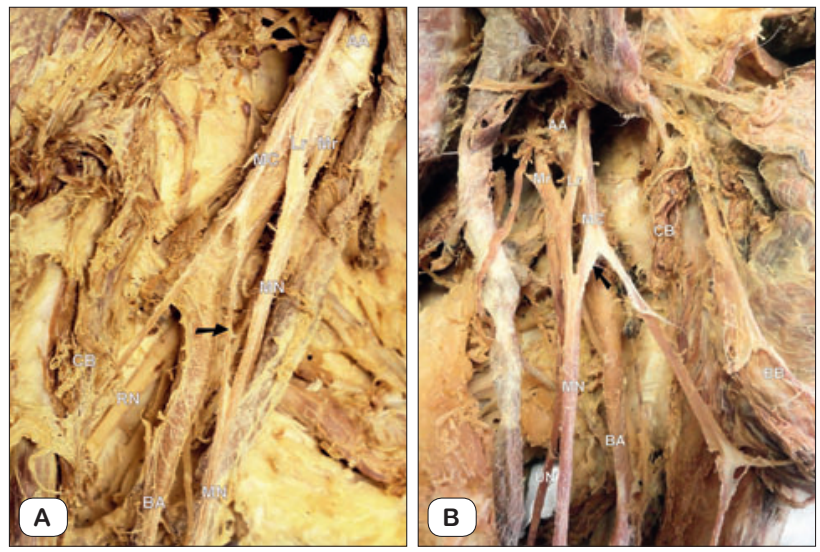

Fig. 2. Proximal communicating branch (black arrow) between musculocutaneous (MC) and median (MN) nerves in right (A) and left (B) upper limbs of adult male cadaver. $\mathrm{Mr}$ - medial root of median nerve, $\mathrm{Lr}$ - lateral root of median nerve, $\mathrm{RN}$ - radial nerve, $\mathrm{UN}$ - ulnar nerve, AA - axillary artery, BA - brachial artery, CB - coraco-

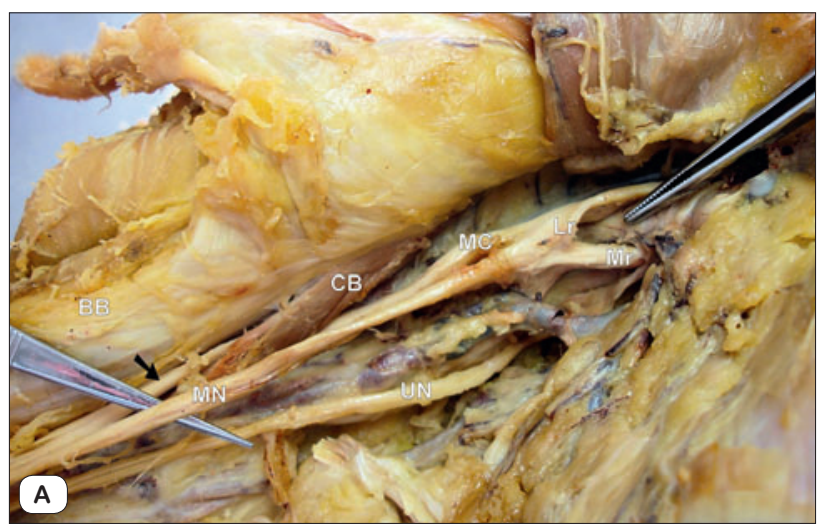
brachialis, BB - biceps brachii.

\section{Methods}

During the routine gross anatomical dissections of the peripheral nerves and vessels, we observed neuronatomical variations in the upper limbs of four formalin embalmed adult cadavers. Musculocutaneous and median nerves were connected by a communicating branch at distinct level in each cadaver.

The axillary fossa, medial bicipital groove, cubital fossa and the anterior antebrachial region were carefully dissected in both upper limbs of each of the four cadavers. The formation manner of musculocutaneous and median nerves, their topographical relations, course and branches were noted in each case to exclude the existence of other anatomical variations. The anomalous connections were measured and later documented by digital camera.

\section{Results}

Variation 1 (Fig. 1). The median nerve was formed in the axillary fossa anterior to the axillary artery by the fusion of the medial and lateral roots. These roots were branches of the corresponding cords of the brachial plexus. A thick branch was separated from

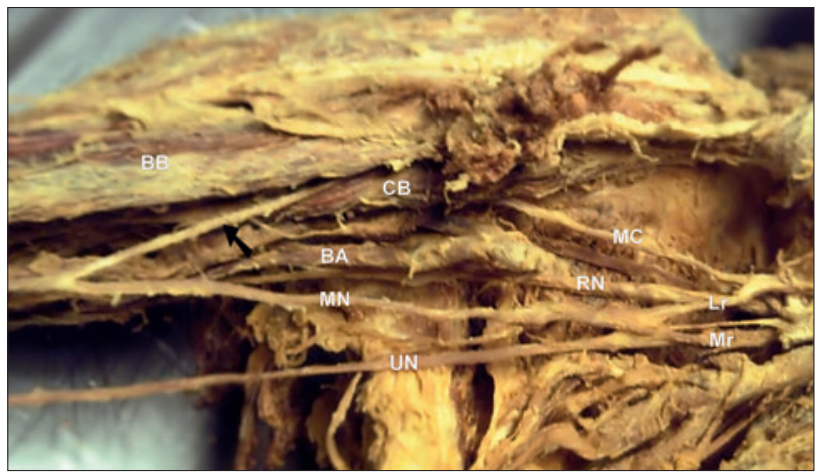

Fig. 3. Unilateral distal communicating branch (black arrow) between musculocutaneous (MC) and median (MN) nerves in male adult cadaver. $\mathrm{Mr}$ - medial root of median nerve, $\mathrm{Lr}$ - lateral root of median nerve, $\mathrm{RN}$ - radial nerve, UN - ulnar nerve, BA-brachial artery, CB - coracobrachialis, BB - biceps brachii.

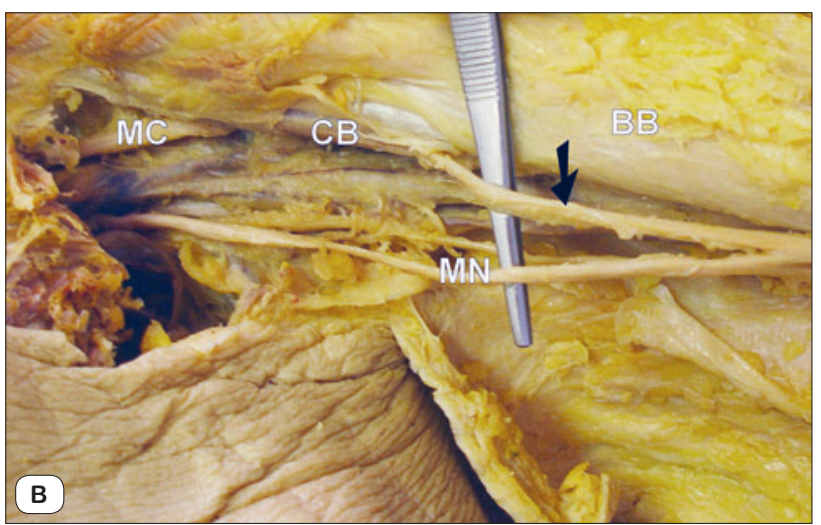

Fig. 4. Distal communicating branch (black arrow) between musculocutaneous (MC) and median (MN) nerves in right (A) and left (B) upper limbs of adult female cadaver. $\mathrm{Mr}$ - medial root of median nerve, $\mathrm{Lr}$ - lateral root of median nerve, $\mathrm{UN}$ - ulnar nerve, $\mathrm{CB}$ - coracobrachialis, BB - biceps brachii. 


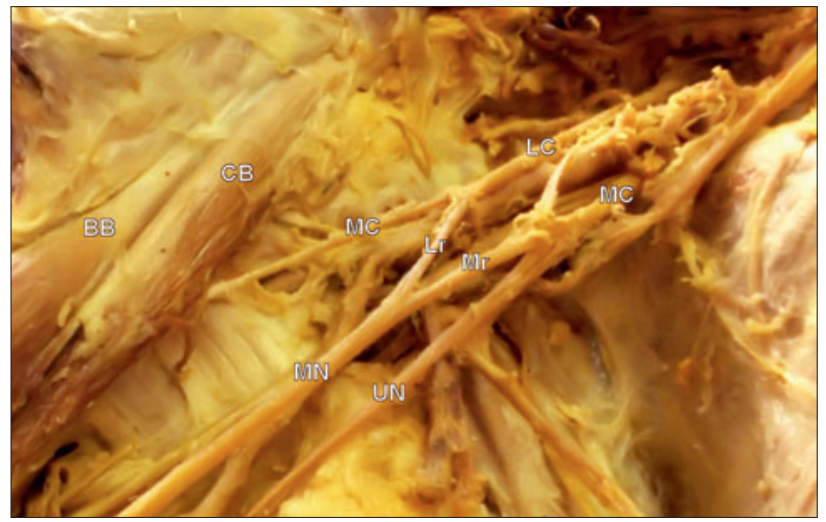

Fig. 5. Main branches of medial (MC) and lateral (LC) cord of the brachial plexus without presence of anatomical variations. $\mathrm{Mr}$ - medial root of median nerve, $\mathrm{Lr}$ - lateral root of median nerve, $\mathrm{MN}$ - median nerve, MC - musculocutaneous nerve, $\mathrm{UN}$ - ulnar nerve, CB - coracobrachialis, BB - biceps brachii.

the musculocutaneous nerve in the axillary fossa about $4 \mathrm{~cm}$ proximally to the nerve piercing point through the coracobrachialis muscle. It coursed medially and distally for about $6.4 \mathrm{~cm}$ and joined the median nerve. The communicating branch was fused with the median nerve medially to the brachial artery and at the level of the lowest insertion point of the pectoralis major muscle. This anomaly was observed unilaterally in the right upper limb of adult male cadaver.

Variation 2 (Figs $2 \mathrm{~A}$ and B). The variable communicating branch was found in both upper limbs of adult male cadaver. It passed obliquely from the musculocutaneous nerve to the median nerve. In right upper limb the communicating branch was split from the musculocutaneous nerve in the axillary fossa about 3.3 $\mathrm{cm}$ proximally to the nerve piercing point through the coracobrachialis muscle and coursed for about $3.8 \mathrm{~cm}$ before it fused with the median nerve. In the left upper limb the communicating branch was separated from the musculocutaneous nerve in the axilla about $3.8 \mathrm{~cm}$ proximally to the nerve piercing point and measured 1.4 $\mathrm{cm}$ at its attachment point with median nerve.

Variation 3 (Fig. 3). The anomalous branch left the musculocutaneous nerve after penetrating the coracobrachialis muscle. It passed downward and medially for about $7.8 \mathrm{~cm}$ to join the median nerve anterior to the brachial artery and at the level of the deltoid muscle insertion. The accessory communicating branch was observed unilaterally in the right upper limb of adult male cadaver.

Variation 4 (Figs 4 A and B). The interconnecting anomaly was detected in both upper limbs of adult female cadaver. The communicating branch originated bilaterally distally to the musculocutaneous nerve penetrating point through the coracobrachialis muscle. It had oblique course and fused with the median nerve approximately at the middle of humerus in both upper limbs. It measured $4.7 \mathrm{~cm}$ in the right upper limb, and $6.3 \mathrm{~cm}$ in the left upper limb.

\section{Discussion}

The musculocutaneous nerve arises from the infraclavicular part of the brachial plexus. The nerve pierces the coracobrachialis after its branching from the lateral cord of the plexus. It descends
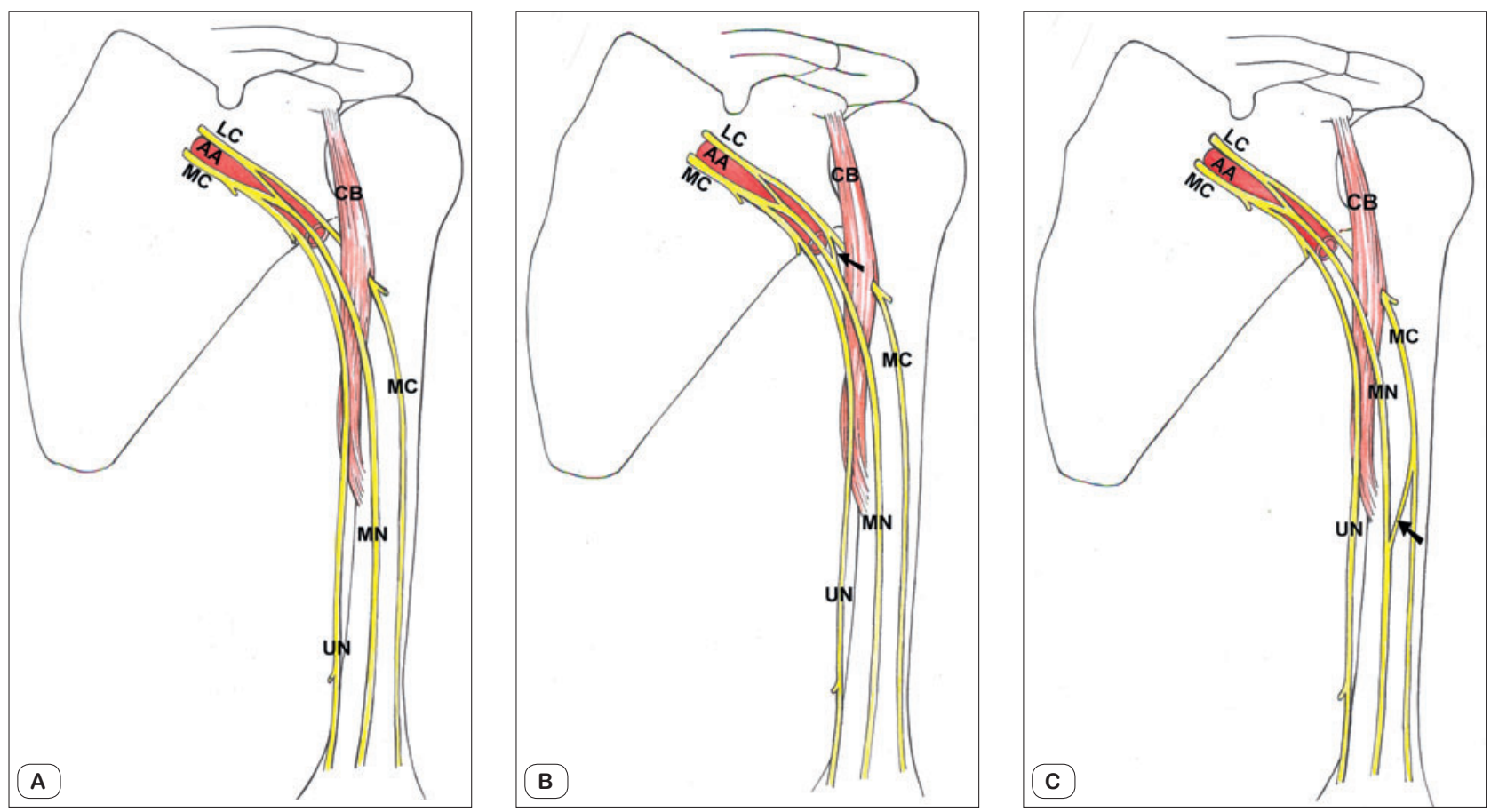

Fig. 6. Scheme of main branches of medial (MC) and lateral (LC) cord of the brachial plexus without presence of anatomical variations (A), with proximal (B) and distal (C) communicating branch (black arrow) between musculocutaneous (MC) and median (MN) nerves. UN - ulnar nerve, AA - axillary artery, CB - coracobrachialis. 
between biceps brachii and brachialis, and at the level of the elbow it continues superficially and laterally as the lateral antebrachial cutaneous nerve. The musculocutaneous nerve innervates the anterior arm muscles and the skin over the lateral surface of the forearm $(9,10)$ (Figs 5, 6 A, B and C).

The median nerve is formed by lateral and medial roots, respectively originates from lateral and medial cords of the brachial plexus. The nerve descends in the medial bicipital groove anterior and lateral to the brachial artery without giving branches to the arm region. It passes in the forearm on the posterior surface of the flexor digitorum superficialis. It enters the palm together with the digits flexors after passing through the carpal tunnel. The median nerve supplies most of the anterior forearm and thenar muscles. Its sensory fibers innervate most skin over the hand palmar surface and, to a limited extent, the dorsal surface of digits $(9,10)$.

The communication between the musculocutaneous and the median nerves is the most common anatomical variation of infraclavicular part of the brachial plexus (11). The appearance frequency of this variable connection was reported to be in the range between 10 and $53.6 \%$ of cases $(12,13,14)$. Many researchers classified the relation between the musculocutaneous and the median nerves. The classification elaborated by Le Minor (1990) is one of the most commonly used in literature. The variations of the musculocutaneous nerve were separated into five types. Type I: Presents the classical description of the musculocutaneous nerve without communication with the median nerve. Type II: Is characterized by fibers connecting both nerves, these communications can be of variable form and level. Type III: The lateral root of the median nerve is originating from the musculocutaneous nerve. Type IV: All fibers of the musculocutaneous nerve are branching from the lateral root of the median nerve. Type V: The musculocutaneous nerve is absent and nerve fibers for anterior arm muscles are branching from the median nerve $(15,16)$.

Applying one of these types on anatomical findings is hard and confusing in many cases. Due to this fact Guerri-Guttenberg and Ingolotti (2009) proposed a classification based on four steps to identify the variation of the musculocutaneous nerve. First step is determining the presence or absence of the nerve. When the nerve is present, the second step determines if the nerve pierces the coracobrachialis muscle. The third step is concentrating on the presence of communication between the median and musculocutaneous nerve. Level of this communication and its relation to the piercing point on the coracobrachialis muscle are solved in the fourth step. The musculocutaneous nerve variations are assigned by numbers and letters at each step (17).

The common criterion of the presented cases is the forming of nerve fibers interconnecting the musculocutaneous and median nerves. The communicating fibers of the variations 1 and 2 were located in the upper third of the arm and proximal to the musculocutaneous nerve penetration through the coracobrachialis muscle. In the variations 3 and 4, the communicating branch was situated in the lower third of the arm and distal to the nerve penetration point.

The relationship of communicating branch with the perforating point of musculocutaneous nerve through the coracobrachialis muscle was proximal in $33-84.6 \%$ and distal in $7.7-65 \%$ of cases in different anatomical studies $(18,19)$.

Evaluation of these anomalies on the base of the Le Minor's classification is leading to conclusion that all four cases can be defined as variations from the type II. The classification according to Le Minor is not considering the level of the interconnecting fibers. The level of formation and the course of the interconnecting fibers have a great rule in the interpretation of their origin. The communicating branch in the upper third of the arm is regarded mostly as an additional lateral root of the median nerve (20). The interconnecting branch in the lower third of arm is considered to be fibers that failed to run through the lateral root, but entered the musculocutaneous nerve and rejoined the median nerve (21).

Guerri-Guttenberg and Ingolotti (2009) are considering the different formation level of the interconnecting fibers in their classification. Accordingly, the variations 1 and 2 are assigned as 1-A-1-P; which means that the musculcutaneous nerve is present, perforating the coracobrachialis muscle, with one communicating branch proximal to the perforating point. The variations 3 and 4 with communicating branch distal to the coracobrachialis muscle are defined as 1-A-1-D.

The embryological development and the factors affecting its progress are the base for interpretation of the anatomical anomalies origin. Circulatory factors or altered signaling between the mesenchymal cells and the neuronal growth cones at the time of brachial plexus forming might be the cause of changes in the nerve arrangement. The effect of these factors is random and can induce different pattern of nerve fusion in each limb, which could cause unilateral or bilateral expression of anatomical variation $(13,20)$.

The upper limb buds are formed in 26-27 days of the embryological life. The brachial plexus appears as a single radicular cone in the upper limb of 34-35 days embryo. The main branches of the plexus are visible by $38-40$ days. The brachial plexus is divided into ventral and dorsal segments. The roots of median and ulnar nerves are arising from the ventral root. The musculcutaneous nerve originates from the median nerve. The common origin of these two nerves could be the explanation of their various anatomical anomalies (1).

The communication between the musculocutaneous and median nerves can be regarded as remnant of the phylogenetic development. Comparative anatomical studies reported observation of similar interconnecting branches in monkeys and apes. The lower vertebrates possess only one nerve trunk in their thoracic limb, which is considered as equivalent to the median nerve (17).

The solitary neuropathies of musculocutaneous nerve are unusual, with trauma being the most common cause. Direct lesions of the nerve can occur as result of humeral fracture, stab or blunt wounds. Dislocation of the shoulder joint and maneuvers used to reduce it are specific causes of multiple nerves trauma involving musculcutaneous, radial, axillary and suprascapular nerves (22).

The non-traumatic musculocutaneous nerve neuropathies are mostly a consequence of nerve compression or traction. The nerve is vulnerable to compression after strenuous physical activity or by chronic pressure secondary to hyperthropy as in weight lifters (17, $23,24)$. The traction neuropathies were reported after sudden or 
violent hyperextension of the upper limb. Stretching of the nerve can be caused also by improper arm position during sleep $(8,25)$.

The clinical picture of the musculocutaneous nerve disorder is related to the level of lesion. Proximal lesions of the nerve are involving its sensory and motor fibers, which causes wasting and weakness of biceps brachii, brachialis and coracobrachialis muscles with sensory disturbances over the radial side of the forearm. The distal lesions cause primarily impairment of the nerve sensory function resulting from pure neuropathy of the lateral cutaneous nerve of the forearm $(22,25)$.

Variable interconnecting branches between musculocutaneous and median nerve have to be considered in the diagnosis of nerve lesions in the axillary and arm regions. Compound musculocutaneous and median nerve neuropathy would occur in lesions of the interconnecting branches (26). Injuries of the musculocutaneous nerve proximal to these branches can cause particular and unexpected clinical symptoms, such as weakness of forearm flexors and thenar muscles (13).

The proximal communicating branch between musculocutaneous and median nerves mostly has close relation with the axillary artery, while the distal branch is related with the brachial artery. This close relation could result in compression of these arteries and impairment of the blood supply to the upper limb $(27,28)$.

\section{References}

1. Tatar I, Brohi R, Sen F, Tonak A, Celik H. Innervation of the coracobrachialis muscle by a branch from the lateral root of the median nerve. Folia Morphol 2004; 63 (4): 503-506.

2. Tubbs RS, Jones VL, Loukas M et al. Anatomy and landmarks for branches of the brachial plexus: a vade mecum. Surg Radiol Anat 2010; 32: 261-270.

3. Johnson EO, Vekris M, Demesticha T, Soucacos PN. Neuroanatomy of the brachial plexus: normal and variant anatomy of its formation. Surg Radiol Anat 2010; 32: 291-297.

4. Mráz P, Belej K, Beňuška J, Holomáňová A, Macková M, Šteňová

J. Anatómia l'udského tela 2. Bratislava; SAP, 2006: 1-487.

5. Shin AY, Spinner RJ, Steinmann SP, Bishop AT. Adult traumatic brachial plexus injuries. J Am Acad Orthop Surg 2005; 13 (6): 382-396.

6. Badawoud MHM. A study on the anatomical variations of median nerve formation. Bahrain Med Bull 2003; 25 (4): 169-171.

7. Zafeiriou DI, Psychogiou K. Obstetrical brachial plexus palsy. Pediatr Neurol 2008; 38: 235-242.

8. Matejčík V. Plexus brachialis et lumbosacralis. Bratislava; Prolitera, 2011: 1-99.

9. Romanes GJ (Ed). Cunningham's textbook of anatomy. 12 $2^{\text {th }}$ ed. Oxford; New York; Toronto; Oxford University Press, 1991: 1-1078.

10. Standring, S (Ed). Gray's Anatomy: the anatomical basis of clinical practice. $39^{\text {th }}$ ed. New York; Churchill Livingstone, 2004: 1-1600.
11. Anyanwu GE, Obikili EN, Esom, Ozoemana FN. Prevalence and pattern of communication of median and musculocutaneous nerves within the black population: Nigeria - a case study. Intern J Biomed Health Sci 2009; 5 (2): 87-94.

12. Das S, Paul S. Anomalous branching pattern of lateral cord of brachial plexus. Int J Morphol 2005; 23 (4): 289-292.

13. Chauhan R, Roy TS. Communication between the median and musculocutaneous nerve - a case report. J Anat Soc India 2002; 51 (1): 72-75.

14. Goyal N, Harjeet, Gupta M. Bilateral variant contribution in the formation of median nerve. Surg Radiol Anat 2005; 27 (6): 562-565.

15. Le Minor JM. A rare variation of the median and musculocutaneous nerves in man. Arch Anat Histol Embryol 1990; 73: 33-42.

16. Kocabiyik N, Yalcin B, Yazar F, Ozan H. An accessory branch of musculocutaneous nerve joining median nerve. Neuroanat 2005; 4: 13-15.

17. Guerri-Guttenberg RA, Ingolotti M. Classifying musculcutaneous nerve variations. Clin Anat 2009; 22: 671-683.

18. Loukas M, Aqueelah H. Musculocutaneous and median nerve connections within, proximal and distal to the coracobrachialis muscle. Folia Morphol 2005; 64 (2): 101-108.

19. Uyaroglu FG, Kayalioglu G, Erturk M. Anastomotic branch from the median nerve to the musculocutneous nerve: a case report. Anat 2008; 2: $63-66$.

20. Satyanarayana N, Vishwakarma N, Kumar GP, Guha R, Datta AK, Sunitha P. Rare variations in the formation of median nerve - embryological basis and clinical significance. Nepal Med Coll J 2009; 11 (4): 287-290.

21. Prasada Rao PV, Chaudhary SC. Communication of the musculocutaneous nerve with the median nerve. East Afr Med J 2000; 77: 498-503.

22. Osborne AWH, Birch RM, Munshi P, Bonney G. The musculocutaneous nerve. Results of 85 repairs. J. Bone Joint Surg 2000; 82-B: 1140-1142.

23. Simonetti S. Musculocutaneous nerve lesion after strenuous physical activity. Muscle Nerve 1999; 22: 647-649.

24. Fattal C, Weber J, Beuret-Blanquart F. Isolated musculocutaneous nerve palsy in a spinal cord injury. Spin Cord 1998; 36: 591-592.

25. Stewart JD, Bourque P. Other mononeuropathies of the upper limbs. 937-953. In: Brown WF, Bolton CF, Aminoff MJ. Neuromuscular function and disease: basic, clinical and electrodiagnostic aspects. Volume 1. Philadelphia; Saunders, 2002.

26. Arora J, Kapur V, Suri RK, Khan RQ. Inter-communications between median and musculocutaneous nerves with dual innervation of brachialis muscle-a case report. J Anat Soc India 2003; 52 (1): 66-68.

27. Fazan VPS, Amadeu AS, Caleffi AL, Rodrigues Filho OA. Brachial plexus variations in its formation and main branches. Acta Cir Bras.2003; 18 (5): $14-18$.

28. Deshmukh AG, Devershi DB. Bilateral variation in the formation of median nerve. J Mahatma Gandhi Inst Med Sci 2006; 11(2): 36-39.

Received August 3, 2011. Accepted January 18, 2013. 